\title{
Prevalence and characteristics of misreporting of energy intake in US children and adolescents: National Health and Nutrition Examination Survey (NHANES) 2003-2012
}

\author{
Kentaro Murakami ${ }^{1 *}$ and M. Barbara E. Livingstone ${ }^{2}$ \\ ${ }^{1}$ Department of Nutrition, School of Human Cultures, University of Shiga Prefecture, Shiga 522-8533, Japan \\ ${ }^{2}$ Northern Ireland Centre for Food and Health, Ulster University, Coleraine BT52 1SA, UK \\ (Submitted 23 June 2015 - Final revision received 10 September 2015 - Accepted 1 October 2015 - First published online 3 November 2015)
}

\section{Abstract}

Using data from the National Health and Nutrition Examination Survey (NHANES) 2003-2012, we investigated the prevalence and characteristics of under- and over-reporting of energy intake (EI) among 14044 US children and adolescents aged 2-19 years. For the assessment of EI, two 24-h dietary recalls were conducted with the use of the US Department of Agriculture Automated Multiple-Pass Method. Under-, plausible and over-reporters of EI were identified using two methods: based on the $95 \%$ confidence limits (1) for agreement between the ratio of EI:BMR and a physical activity level for sedentary lifestyle (1.55) and (2) of the expected ratio of EI:estimated energy requirement (EER) of 1·0. BMR was calculated using Schofield's equations. EER was calculated using equations from the US Dietary Reference Intakes, assuming 'low active' level of physical activity. The risk of being an under- or over-reporter compared with a plausible reporter was analysed using multiple logistic regression. Percentages of under-, plausible and over-reporters were $13 \cdot 1,81 \cdot 5$ and $5.4 \%$, respectively, based on EI:BMR and $18.8,72.3$ and $8.8 \%$, respectively, based on EI:EER. Under-reporting was associated with older age, non-Hispanic blacks (compared with non-Hispanic whites) and overweight and obesity (compared with normal weight). Over-reporting was associated with younger age, lower family poverty income ratio, normal weight and the first survey cycle. Similar findings were obtained when analysing only the first 24-h recall data from NHANES 1999-2012 ( $n$ 22 949). In conclusion, we found that EI misreporting remains prevalent and differential in US children and adolescents.

\section{Key words: Energy intake: Misreporting: Children: National Health and Nutrition Examination Survey}

Misreporting of dietary intake is a common phenomenon that appears to occur both randomly and non-randomly ${ }^{(1-3)}$ and may be selective for different kinds of foods and nutrients ${ }^{(4,5)}$ The resulting potential for differential errors in dietary data complicates the interpretation of studies on diet and health and, at worst, might produce spurious diet-health relations ${ }^{(1,3,5)}$. Thus, the identification of the characteristics associated with misreporting (under- and over-reporting) of dietary intake is important to increase the understanding of this issue.

The measurement of dietary intake in children and adolescents is an integral component for monitoring the nutritional status of these age groups and for conducting epidemiological and clinical research on the links between diet and health. However, it is made particularly challenging by the many unique respondent and observer considerations that surface at different ages from early childhood to late adolescence, including cognitive abilities and dietary habits ${ }^{(6,7)}$. Nevertheless, investigations of the misreporting of energy intake (EI), a surrogate measurement of the total quantity of food intake, in children and adolescents are limited ${ }^{(1,6,7,8-23)}$. It is conceivable that the way in which survey participants comply with dietary assessment procedures may differ by country and over time.

In the continuous National Health and Nutrition Examination Survey (NHANES), the US Department of Agriculture (USDA) Automated Multiple-Pass Method is used for collecting 24-h dietary recall information. Although this method has been validated against total energy expenditure measured by doubly labelled water ${ }^{(24,25)}$ and against observed actual intake ${ }^{(26,27)}$ in adult populations, the validity in children and adolescents is largely unknown. In the current study, the prevalence and characteristics of under- and over-reporting of EI among US children and adolescents were evaluated using data from the NHANES.

Abbreviations: EER, estimated energy requirement; EI, energy intake; NHANES, National Health and Nutrition Examination Survey; PAL, physical activity level; USDA, US Department of Agriculture.

* Corresponding author: Dr K. Murakami, fax +81 74949 8499, email kenmrkm@m.u-tokyo.ac.jp 


\section{Methods}

\section{Survey design}

The present cross-sectional analysis was based on public domain data from NHANES, a continuing population-based survey that uses a complex, stratified multistage probability sample design to create a representative sample of the noninstitutionalised civilian US population ${ }^{(28,29)}$. Beginning in 1999, the survey examines about 5000 persons each year and the data are released every 2 years. Each survey consists of questionnaires administered in the home, followed by a standardised health examination, including an in-person 24-h dietary recall interview, in a mobile examination centre. Since 2002, a second 24-h dietary recall was also obtained by telephone; two 24-h dietary recall data are publicly available since 2003. The unweighted response rates for the examined persons aged 1-19 years for NHANES 1999-2000, 2001-2002, 2003-2004, 2005-2006, 2007-2008, 2009-2010 and 2011-2012 were $84,87,84,84,83,86$ and $77 \%$, respectively ${ }^{(30)}$. The documentation and data for each of these surveys can be downloaded from the NHANES website ${ }^{(31)}$. The NHANES was conducted according to the guidelines laid down in the Declaration of Helsinki, and all procedures involving human subjects were approved by National Center for Health Statistics Research Ethics Review Board. Written informed consent was obtained from all subjects or from their proxies.

\section{Analytic sample}

The analytic sample was limited to children and adolescents aged 2-19 years with two complete and reliable 24-h dietary recall data ( $n$ 15279). After excluding pregnant ( $n$ 96) and lactating ( $n$ 15) respondents as well as those with missing information on the variables of interest (family income: $n$ 882; body height or weight: $n$ 242), the final analytic sample included 14044 respondents from NHANES 2003-2012. An additional analysis was also conducted using only the first dietary recall data in 22949 respondents from NHANES 1999-2012. There were no significant differences in EI misreporting variables between the subjects included in the analysis and those excluded from the analysis (data not shown).

\section{Assessment of energy intake}

All surveys collected dietary information with the use of a 24-h dietary recall administered by a trained interviewer in the mobile examination centre. Beginning with 2002, a second 24-h dietary recall was also obtained via telephone $3-10 \mathrm{~d}$ after the first recall. The dietary recalls collected for the NHANES 1999-2000 and 2001 survey years used a computer-assisted interview that included a four-step multiple-pass approach. Since 2002, the dietary data were collected with the use of an automated five-step multiple-pass approach, namely the USDA Automated Multiple-Pass Method ${ }^{(24-27,31)}$. This method consists of the following: (1) a quick list pass, in which the respondent is asked to list everything eaten or drunk the previous day; (2) a forgotten foods list pass, in which a standard list of foods or beverages - often forgotten - is read to prompt recall; (3) a time and occasion pass, in which the time of and the name for the eating occasion are collected; (4) a detail and review pass, in which detailed descriptions and portion sizes are collected and the time interval between meals is reviewed to check for additional foods; and (5) the final probe pass, one last opportunity to remember foods consumed. Proxies, most commonly a parent, reported dietary intake for children aged $2-5$ years and assisted with the dietary interview for children aged 6-11 years; dietary intake was self-reported by adolescents aged 12-19 years. Estimates of EI from all reported foods and beverages were calculated using the USDA food composition databases. In 1999-2000, the USDA 1994-1998 Survey Nutrient Database was the food composition database used; in subsequent surveys, the USDA Food and Nutrient Database for Dietary Studies was used ${ }^{(31)}$. The average of EI over the $2 \mathrm{~d}$ for each participant was used for the present analysis.

\section{Assessment of non-dietary variables}

Consistent with NHANES sample-selection methods, age was categorised as 2-5, 6-11 and 12-19 years. Race/ethnicity was categorised as non-Hispanic white, non-Hispanic black, Mexican American and others. As an indicator of socioeconomic status, we used family income as a percentage of the federal poverty threshold, which was categorised as $<130,130-349$ and $\geq 350 \%$. The hours of screen time were determined from questions on television/video watching $(\mathrm{h} / \mathrm{d})$ or computer use (h/d) over the past $30 \mathrm{~d}$ (except for adolescents aged 12-19 years in NHANES 2007-2008 and 2009-2010 for which information on sedentary activity was used), which were categorised as $<2, \geq 2$ to $<4, \geq 4$ to $<6$ and $\geq 6 \mathrm{~h} / \mathrm{d}$. Body weight and height were measured by trained interviewers using standardised procedures with calibrated equipment. BMI $\left(\mathrm{kg} / \mathrm{m}^{2}\right)$ was calculated as weight $(\mathrm{kg})$ divided by height squared $\left(\mathrm{m}^{2}\right)$. The percentile of BMI for age was calculated using the SAS program for growth charts available from the Centers for Disease Control and Prevention ${ }^{(32,33)}$. Weight status was defined on the basis of the percentile of BMI for age as follows ${ }^{(34)}$ : underweight $(<5$ th percentile), normal $(\geq 5$ th to $<85$ th percentile), overweight ( $\geq 85$ th to $<95$ th percentile) and obese ( $\geq 95$ th percentile).

\section{Evaluation of the accuracy of energy intake reporting}

Misreporting of EI was evaluated on the basis of the ratio of EI:BMR (the Goldberg's cut-off) ${ }^{(35)}$ and the ratio of EI:estimated energy requirement (EER), namely the procedure proposed by Huang et $a l .{ }^{(36)}$. Subjects were identified as plausible, underand over-reporters of EI according to whether the individual's ratio was within, below or above the $95 \%$ confidence limits for agreement between EI:BMR and the respective physical activity level (PAL) or of the expected EI:EER of 1.0. For the principles of the Goldberg's cut-off, the PAL for sedentary lifestyle (i.e. 1.55) ${ }^{(35)}$ was applied for all subjects, because of a lack of an objective measure of physical activity in the present study. BMR was estimated using Schofield's sex- and age-specific equations based on body height and weight ${ }^{(37)}$. The $95 \%$ confidence limits for agreement (upper and lower cut-off values) between 
EI:BMR and the PAL were calculated, taking into account CV in intakes and other components of energy balance (i.e. the within-subject variation in EI: $23 \%$; the precision of the estimated BMR relative to the measured BMR: $8.5 \%$; the between-subject variation in PAL: $15 \%)^{(35)}$. Consequently, under-, plausible and over-reporters were defined as having EI: BMR of $<0.96,0.96-2.49$ and $>2.49$ for $2 \mathrm{~d}$ data and $<0.87$, $0.87-2.75$ and $>2.75$ for $1 \mathrm{~d}$ data, respectively.

EER was calculated using sex-, age- and weight status-specific equations published in the US Dietary Reference Intakes, based on sex, age, body height and weight and physical activity ${ }^{(38)}$. Because of a lack of an objective measure of physical activity as mentioned above, we assumed 'low active' level of physical activity (i.e. PAL $\geq 1.4$ to $<1 \cdot 6)^{(38)}$ for all subjects during this calculation. The $95 \%$ confidence limits of the expected EI:EER ratio of 0 on the natural $\log$ scale were calculated, taking into account $\mathrm{CV}$ in intakes and other components of energy balance (i.e. the within-subject variation in EI: $23 \%$; the error in the EER equations: $4.8 \%$; the day-to-day variation in total energy expenditure: $8 \cdot 2 \%)^{(36)}$. Consequently, under-, plausible and over-reporters were defined as having EI:EER of $<0.69$, $0.69-1.46$ and $>1.46$ for $2 \mathrm{~d}$ data and $<0.61,0.61-1.64$ and $>1.64$ for $1 \mathrm{~d}$ data, respectively.

\section{Statistical analysis}

Statistical analyses were performed using SAS statistical software (version 9.2; SAS Institute). All reported $P$ values are two-tailed, and $P<0.01$ was considered statistically significant to reduce the likelihood of making a type 1 error. All of the analyses used the NHANES-provided sampling weights that were calculated to take into account unequal probabilities of selection resulting from the sample design, non-response and planned oversampling of selected subgroups, so that the results are representative of the US community-dwelling population $^{(29,39)}$. For EI, BMR, EER, EI:BMR and EI:EER, sample-weighted means (with their standard errors) were generated using PROC SURVYMEANS procedure. Differences in these variables across categories of each of the characteristics were examined by Wald's $F$ test using PROC SURVEYREG procedure. Proportions (with their standard errors) of under-, plausible and over-reporters of EI were calculated using PROC SURVEYFREQ procedure. Differences in proportions of under-, plausible and over-reporters across categories of each of the characteristics were examined by $\chi^{2}$ test using PROC SURVEYFREQ procedure.

The risk of being classified as an under-reporter of EI compared with being a plausible reporter or as an over-reporter compared with being a plausible reporter, was estimated using logistic regression. First, with the use of PROC SURVEYLOGISTIC procedure, crude OR and 99\% CI for the risk of being classified as an under- or over-reporter were calculated for each category of factors that are possibly associated with EI misreporting, namely sex (reference: boys), age group (reference: 2-5 years), race/ethnicity (reference: non-Hispanic white), family poverty income ratio (reference: <130\%), weight status (reference: normal), watching television and computer use (reference: $<2 \mathrm{~h} / \mathrm{d}$ ) and survey cycle (reference: 2003-2004).
Multivariate-adjusted OR and 99\% CI were then calculated by entering all variables simultaneously into the regression model to assess the independent associations.

These analyses were conducted for boys and girls separately. The results on the association between EI reporting and the variables examined were essentially the same in boys and girls. The following six interaction terms were also examined: age by sex, age by weight status, age by survey cycle, sex by weight status, sex by survey cycle and weight status by survey cycle. There was an interaction between age and sex for EI:BMR $(P=0.0005)$, an interaction between age and weight status for EI:BMR and EI:EER (both $P<0.0001$ ) and an interaction between sex and weight status for EI:EER $(P=0 \cdot 001)$. Multivariate analyses stratified by sex (boys and girls), age group (2-5, 6-11 and 12-19 years) or weight status (underweight and normal weight combined and overweight and obese combined), however, provided similar findings (except for no association of weight status with both under- and over-reporting based on EI:BMR in subjects aged 2-5 years and female sex associated with a lower risk of over-reporting based on EI:BMR in subjects aged 12-19 years). The present paper thus presents the results for the whole analytic sample combined.

\section{Results}

Among 14044 subjects with $2 \mathrm{~d}$ dietary data, the sampleweighted mean EI:BMR was 1.55, whereas the corresponding value for EI:EER was 0.99 (Table 1). Boys had a higher mean EI: BMR than that of girls. Mean EI:BMR differed significantly among age groups, with the highest in the youngest group (2-5 years) and the lowest in the oldest group (12-19 years), among race/ethnicity groups, with the lowest in non-Hispanic blacks and among survey cycles, with the highest in 2003-2004 and the lowest in 2007-2008. Mean EI:BMR in obese and overweight subjects was lower compared with that in normalweight and underweight subjects. Watching television and computer use was inversely associated with EI:BMR. Similar associations of these characteristics with EI:EER were also observed. Multiple regression analyses showed that $22 \%$ of the variance in EI:BMR and $21 \%$ of the variance in EI:EER were explained by the variables examined here (i.e. sex, age group, race/ethnicity, family poverty income ratio, weight status, watching television and computer use and survey cycle).

The sample-weighted percentages of under-, plausible and over-reporters of EI were $13 \cdot 1,81.5$ and $5 \cdot 4 \%$, respectively, on the basis of EI:BMR and $18 \cdot 8,72.3$ and $8.8 \%$, respectively, on the basis of EI:EER (Table 2). Using EI:BMR, the percentages of under-reporters and over-reporters did not differ between boys and girls. With regard to age, there were more under-reporters among the oldest group, whereas there were more overreporters among the youngest group. For race/ethnicity, there were more under-reporters in non-Hispanic blacks and fewer over-reporters in non-Hispanic whites. The family poverty income ratio was inversely associated with the percentages of both under- and over-reporters. There were more underreporters and fewer over-reporters among overweight and 
Table 1. Characteristics of the subjects: National Health and Nutrition Examination Survey $2003-2012$ ( $n$ 14 044)*

(Numbers and percentages; mean values with their standard errors)

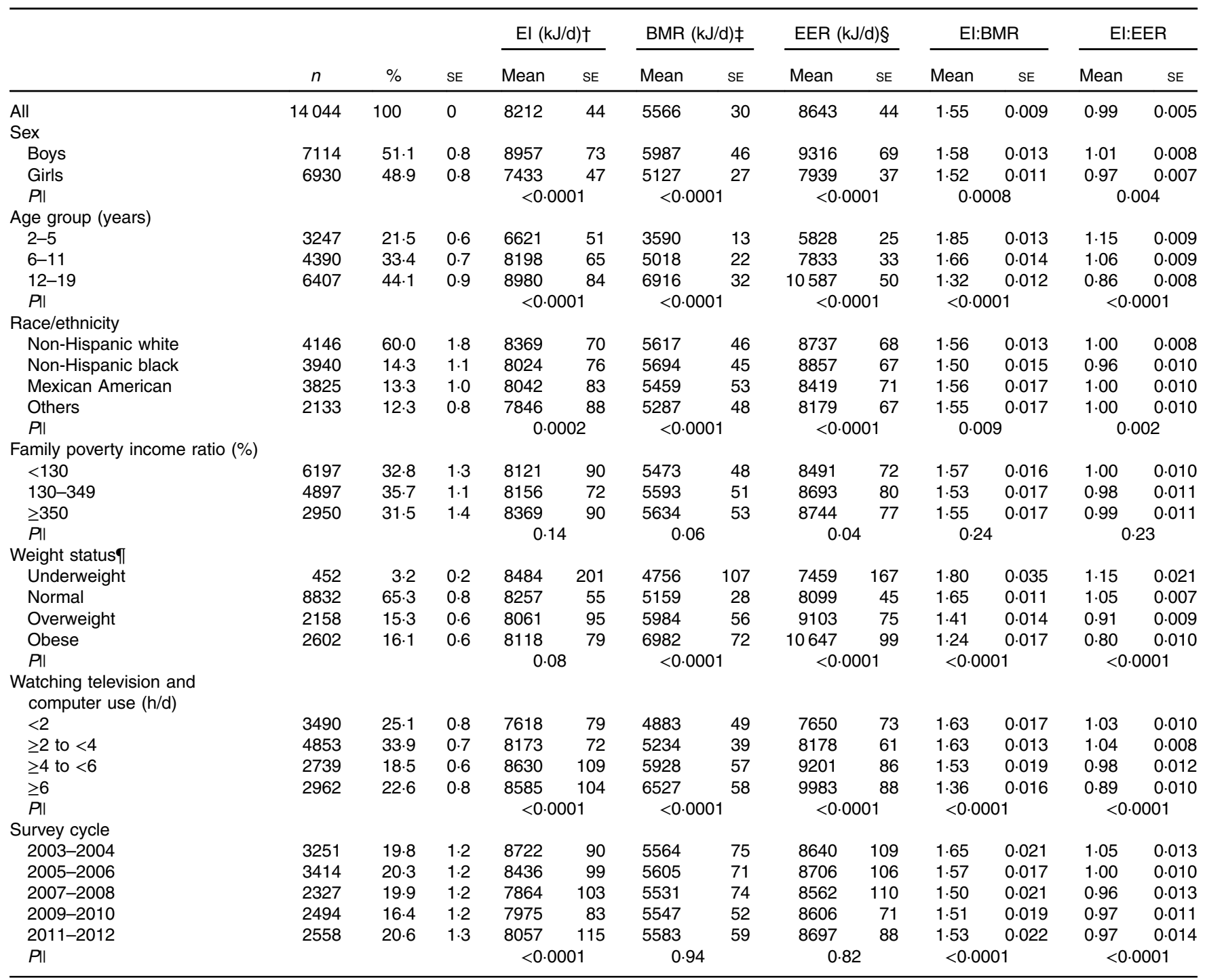

El, energy intake; EER, estimated energy requirement.

* All percentages and mean values are weighted to reflect the survey design characteristics. Analyses are based on subjects with complete data on two $24-\mathrm{h}$ dietary recalls as well as complete information on the variables of interest.

$\dagger$ Based on average values of two 24-h dietary recalls.

$\ddagger$ Estimated using Schofield's sex- and age-specific equations based on body height and weight ${ }^{(34)}$.

$\S$ Calculated using sex-, age- and weight status-specific equations from the US Dietary Reference Intakes based on sex, age and body height and weight assuming 'low active' level of physical activity for all subjects ${ }^{(35)}$.

॥ Based on Wald's $F$ test.

II Defined based on the percentile of BMI for age: $<5$ th percentile for underweight, $\geq 5$ th to $<85$ th percentile for normal, $\geq 85$ th to $<90$ th percentile for overweight and $>95$ th percentile for obese subjects ${ }^{(31)}$.

obese subjects. Longer hours of television watching and computer use were associated with higher percentage of under-reporters and lower percentage of over-reporters. The proportion of under- and over-reporters differed among survey cycles, with more under-reporters in 2007-2008 and more over-reporters in 2003-2004. The results were similar on the basis of using EI:EER to estimate misreporters.

OR and $99 \%$ CI for the risk of being an under-reporter compared with a plausible reporter are shown in Table 3. The results for the crude and multivariate-adjusted models were generally similar except for watching television and computer use. In the multivariate analyses, on the basis of EI:BMR and EI:EER, a higher risk of being an under-reporter was associated with ages $6-11$ years (EI:BMR only) and 12-19 years (compared with age $2-5$ years), non-Hispanic blacks (compared with non-Hispanic white), overweight and obesity (compared with normal weight) and the survey cycle 2007-2008 (EI:BMR only) (compared with 2003-2004). A lower risk of being an under-reporter was associated with underweight (EI:EER only).

Table 4 lists OR and $99 \%$ CI for the risk of being an overreporter compared with a plausible reporter. The results for the 


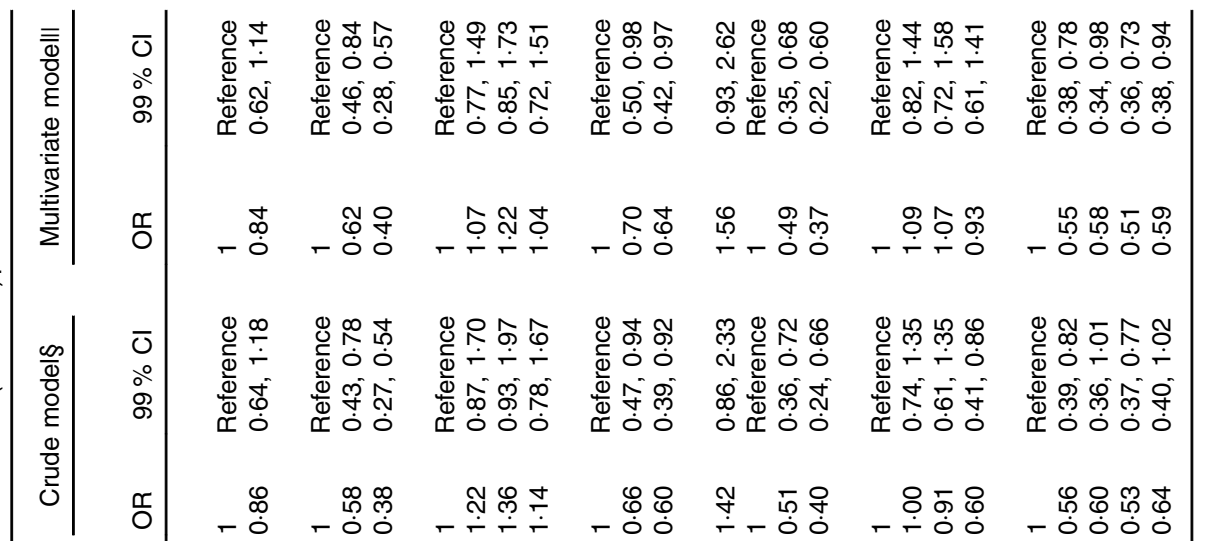

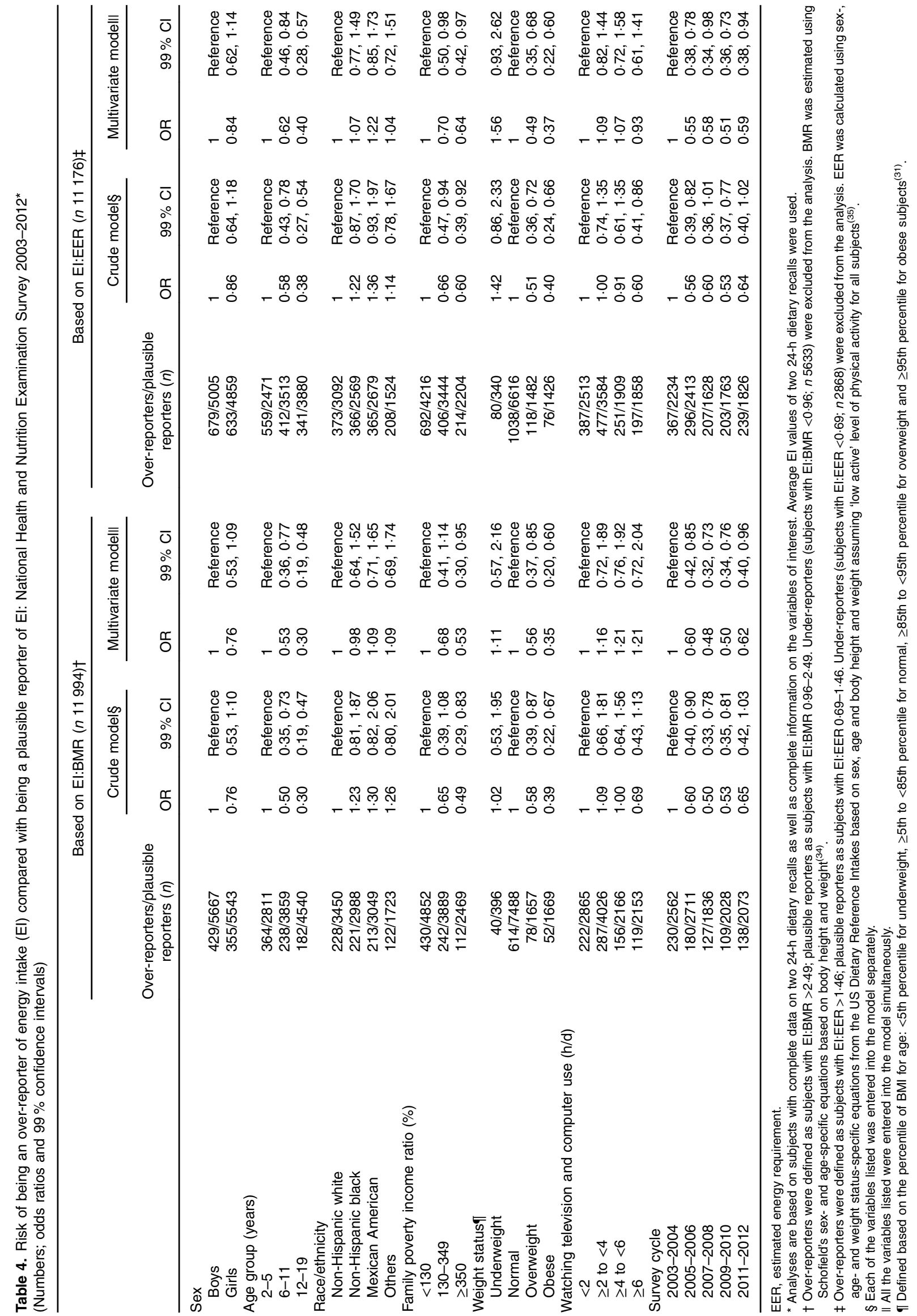


crude and multivariate-adjusted models were again generally similar. In multivariate analyses, a lower risk of being an overreporter was associated with ages 6-11 and 12-19 years, higher family poverty income ratio, overweight and obese and all later survey cycles.

We repeated all the analyses using 22949 subjects with the first dietary recall data. The sample-weighted mean EI:BMR was 1.59, whereas the corresponding value for EI:EER was 1.02 (online Supplementary Table S1). The sample-weighted percentages of under-, plausible and over-reporters of EI were $11 \cdot 6,83.0$ and $5.4 \%$, respectively, on the basis of EI:BMR and $14.7,77.9$ and $7.3 \%$, respectively, on the basis of EI:EER (online Supplementary Table S2). Factors significantly associated with the risk of being an under-reporter or being an over-reporter compared with being a plausible reporter were generally similar (online Supplementary Tables S3 and S4, respectively), except for girls having a higher risk of being an under-reporter and having a lower risk of being an over-reporter.

\section{Discussion}

Using two 24-h dietary recall data from NHANES 2003-2012, we found that misreporting of EI was prevalent and differential in US children and adolescents aged 2-19 years. Percentages of under- and over-reporters of EI were $13 \cdot 1$ and $5.4 \%$, respectively, on the basis of EI:BMR and 18.8 and $8.8 \%$, respectively, on the basis of EI:EER. A higher risk of being an under-reporter of EI compared with being a plausible reporter was associated with older age, non-Hispanic blacks (compared with non-Hispanic whites) and overweight and obesity (compared with normal weight). A higher risk of being an over-reporter compared with being a plausible reporter was associated with younger age, lower family poverty income ratio, normal weight and the first survey cycle. Similar findings were observed when analysing based on the first 24-h dietary recall only (NHANES 1999-2012). To our knowledge, this is the first study to examine the prevalence and characteristics of misreporting of EI in a representative sample of US children and adolescents.

Only a few national studies have examined the misreporting of EI among children and adolescents. A French study evaluated the EI assessed using a 7-d diet record among 1455 boys and girls aged 3-17 years on the basis of the Goldberg's principles ${ }^{(16)}$. The prevalence of under- and over-reporters was 4.9 and $1.4 \%$ for children aged $3-10$ years and 26.0 and $0 \%$ for adolescents aged 11-17 years, respectively. In Australian boys and girls aged $2-16$ years ( $n$ 4800), the EI estimated using a 24-h dietary recall was similarly evaluated, and the prevalence of under- and over-reporters was 5.0 and $3.0 \%$, respectively ${ }^{(17)}$. Additionally, the prevalence of under-reporters of EI (obtained from a 24 -h dietary recall) was $7 \%$ in 653 children aged 3-12 years and $32 \%$ in 517 adolescents aged 13-18 years in Greece (over-reporters not defined) $^{(21)}$. Among 1636 boys and girls aged 4-18 years in Britain, the EI assessed using a 7-d weighed dietary record was evaluated according to EI:EER ${ }^{(18)}$. The prevalence of under- and over-reporters was 19 and $0.7 \%$, respectively, for children aged 4-10 years and 52 and $0.4 \%$, respectively, for adolescents aged 11-18 years. In this analysis based on NHANES, the prevalence of under-reporting $(13 \cdot 1 \%$ on the basis of EI:BMR and $18.8 \%$ on the basis of EI:EER) was within the range of those observed in other countries, whereas the prevalence of over-reporting $(5.4 \%$ on the basis of EI:BMR and $8.8 \%$ on the basis of EI:EER) was somewhat higher. Although it is difficult to determine whether the difference in the prevalence among countries reflects the true difference in the accuracy of reporting or is merely due to differences in the criteria used to identify misreporters, dietary assessment instruments, food composition databases and population characteristics, these national studies clearly show that misreporting of EI is a serious problem in dietary surveys among children and adolescents.

In the present study, overweight and obese subjects were more likely to under-report EI, which has been consistently observed in many studies ${ }^{(8,10,11,16,22)}$. Additionally, older age was associated with under-reporting of EI, which is again consistent with previous studies ${ }^{(12,13,22,36)}$. In adolescents, the additional demands imposed on reporting by increased energy requirements, unstructured eating patterns, a significant degree of out-of-home eating, concerns with self-image and rebellion against authority may contribute to poor compliance in a dietary assessment ${ }^{(7)}$, resulting mainly in dietary under-reporting. For other correlates of misreporting, research is limited or the results are generally inconsistent ${ }^{(1)}$. For race/ethnicity, we found that a higher risk of under-reporting was associated with non-Hispanic blacks (compared with non-Hispanic whites), which had also been observed among US adults from NHANES $1988-1991^{(40)}$. In the present study, sex was not associated with EI misreporting. Several previous studies have found a similar degree of misreporting of EI in boys and girls ${ }^{(8,12,22)}$, although there is some evidence that under-reporting is more prevalent in girls than in boys ${ }^{(1,18,19)}$. Characteristics associated with over-reporting of EI are less understood. We found that over-reporting was associated with younger age, lower family poverty income ratio and normal weight. One study has shown that over-reporting is prevalent at a younger age ${ }^{(36)}$. In an analysis of Irish adults, lower social class was associated with a higher risk of over-reporting ${ }^{(41)}$. Although these variables may not always be associated with EI misreporting, and the association should be dependent on the population characteristics, dietary assessment methods and the procedure for identifying misreporters, the accumulating literature clearly indicates that misreporting occurs non-randomly in children and adolescents. Specific to NHANES, we found that survey cycle was associated with both under- and over-reporting of EI. Although this observation may be explained, at least partly, by the survey design (such as oversampling of non-Hispanic black persons in NHANES 2007-2012), this differential reporting may severely distort the validity of trend analyses using dietary intake data. Thus, previous trend analyses should be cautiously interpreted in this regard, and future analyses should properly take into account misreporting of EI. Alternatively, given the decline in food and beverage purchases in the 2003-2011 period, especially in households with children ${ }^{(42)}$, combined with the most recent statistics about obesity indicating no increase or decrease ${ }^{(43-45)}$, the decline in EI in the later years observed in this study may not totally be misreporting but a true trend ${ }^{(46)}$. 
Several limitations of the present study are acknowledged. At present, the only way to obtain unbiased information on energy requirements in free-living settings is to use doubly labelled water as a biomarker. This technique is expensive and impractical for the application to large-scale epidemiological studies, and thus alternative procedures are used $^{(3,5,8-10,12,15-19,35,36)}$. In the present study, EER was calculated with the use of equations from the US Dietary Reference Intakes, which have been developed based on a large number of measurements of total energy expenditure by the doubly labelled water method and are highly accurate $\left(R^{2} \geq 0 \cdot 95\right)^{(38)}$. In the absence of actual, measured total energy expenditure, these equations should serve as the best proxy. Because of constraints within the data set, we did not have a validated and individualised measure of physical activity. Instead, we assumed 'low active' level of physical activity for all subjects in the calculation of EER (as well as using the PAL for sedentary lifestyle for all subjects when using the Goldberg's principles). This seems adequate for most US children and adolescents, based on accelerometer data in NHANES 2003-2006 (47,48). Nevertheless, in very active individuals (e.g. those aged 6-11 years, as has been reported) ${ }^{(48)}$, EER would be underestimated, resulting in an overestimation of EI:EER and retention of those in plausible or over-reporters. We conducted additional analyses where we assumed 'active' level of physical activity (i.e. PAL $\geq 1.6$ to $<1.9)^{(35)}$ for subjects aged 6-11 years during the calculation of EER as well as using the PAL for active lifestyle $(1.75)^{(35)}$ when using the Goldberg's cut-off. Compared with the original analysis, the percentage of under-reporters ( $14.5 \%$ on the basis of EI:BMR and $21.0 \%$ on the basis of EI: EER) was higher, whereas that of over-reporters $(4.1 \%$ on the basis of EI:BMR and $6.8 \%$ on the basis of EI:EER) was lower, with that of plausible reporters ( $81.4 \%$ on the basis of EI:BMR and $72.1 \%$ on the basis of EI:EER) being unchanged. However, factors significantly associated with the risk of being an underreporter or being an over-reporter compared with being a plausible reporter were generally similar (data not shown). Further, we do not know the sensitivity and specificity of the procedures for identifying under- and over-reporters of EI used; additionally, there is currently not enough information on relative merits of the different methods (i.e. EI:BMR and EI:EER) for detecting misreporters, although EI:EER may be better given that the magnitude of misreporting can be estimated without information on the exact PAL value. Thus, we are unable to determine whether the associations found between misreporting of EI and several characteristics are true, or were artifacts caused by the procedure used to identify misreporters, as well as errors associated with food composition databases used and differences in the dietary interview methodology (i.e. a four-step multiple-pass approach in NHANES 1999-2000 and 2001 survey years $v$. an automated five-step multiple-pass approach in subsequent survey years). Finally, the crosssectional nature of the study does not permit the assessment of causality, owing to the uncertain temporality of the association.

In conclusion, in this comprehensive analysis based on data from NHANES 2003-2012, we found that misreporting of EI assessed using two 24-h dietary recalls was too prevalent to ignore in US children and adolescents aged 2-19 years: $18.5 \%$ on the basis of EI:BMR and $27.7 \%$ on the basis of EI:EER. More importantly, such EI misreporting was differential among populations. Under-reporting was associated with older age, non-Hispanic blacks (compared with non-Hispanic whites) and overweight and obesity (compared with normal weight), whereas over-reporting was associated with younger age, lower family poverty income ratio, normal weight and the first survey cycle. The results were similar when only the first 24-h dietary recall was assessed based on data from NHANES 1999-2012. Thus, it is essential to consider this differential misreporting of EI when investigating diet-disease associations or conducting trend analyses based on NHANES. For example, it may be useful to examine whether the dietary variable of interest is associated with EI misreporting and, if so, to conduct analyses including and excluding EI misreporters or those with and without adjustment for EI misreporting ${ }^{(3,5,18,36,49)}$ so that the influence of EI misreporting on the research results can be acknowledged. For practice, the routine application of some procedures to identify and separately treat those who report data of poor validity would improve the precision and accuracy of results from dietary surveys. As differential misreporting of EI is the inevitable end result of differential misreporting of foods, future research is needed to understand which foods are misreported (in addition to the development of biomarkers suitable for food intake) and, more fundamentally, why people misreport food intake (with the development of a conceptual framework of misreporting) so that dietary assessments are to be improved.

\section{Acknowledgements}

This work was supported in part by the Grants-in-Aid for Young Scientists (B) from the Ministry of Education, Culture, Sports, Science and Technology of Japan (K. M., grant number 15K16213). The Ministry of Education, Culture, Sports, Science and Technology of Japan had no role in the design, analysis or writing of this article.

K. M. contributed to the concept and design of the study, statistical analysis, data interpretation and manuscript writing. M. B. E. L. critically reviewed the manuscript. All authors read and approved the final manuscript.

The authors declare that there are no conflicts of interest.

\section{Supplementary material}

For supplementary material/s referred to in this article, please visit http://dx.doi.org/doi:10.1017/S0007114515004304

\section{References}

1. Livingstone $\mathrm{MBE}$ \& Black AE (2003) Markers of the validity of reported energy intake. J Nutr 133, Suppl. 3, 895S-920S

2. Tooze JA, Subar AF, Thompson FE, et al. (2004) Psychosocial predictors of energy underreporting in a large doubly labeled water study. Am J Clin Nutr 79, 795-804. 
3. Mattisson I, Wirfalt E, Aronsson CA, et al. (2005) Misreporting of energy: prevalence, characteristics of misreporters and influence on observed risk estimates in the Malmo Diet and Cancer cohort. Br J Nutr 94, 832-842.

4. Poppitt SD, Swann D, Black AE, et al. (1998) Assessment of selective under-reporting of food intake by both obese and non-obese women in a metabolic facility. Int $J$ Obes Relat Metab Disord 22, 303-311.

5. Rosell MS, Hellenius MLB, De Faire UH, et al. (2003) Associations between diet and the metabolic syndrome vary with the validity of dietary intake data. Am J Clin Nutr $\mathbf{7 8}$, 84-90.

6. Livingstone MBE \& Robson PJ (2000) Measurement of dietary intake in children. Proc Nutr Soc 59, 279-293.

7. Livingstone MBE, Robson PJ \& Wallace JMW (2004) Issues in dietary intake assessment of children and adolescents. Br J Nutr 92, Suppl. 2, S213-S222.

8. Vagstrand K, Lindroos AK \& Linne Y (2009) Characteristics of high and low energy reporting teenagers and their relationship to low energy reporting mothers. Public Health Nutr $\mathbf{1 2}$, 188-196.

9. Lanctot JQ, Klesges RC, Stockton MB, et al. (2008) Prevalence and characteristics of energy underreporting in AfricanAmerican girls. Obesity 16, 1407-1412.

10. Ventura AK, Loken E, Mitchell DC, et al. (2006) Understanding reporting bias in the dietary recall data of 11-year-old girls. Obesity 14, 1073-1084.

11. Fisher JO, Johnson RK, Lindquist C, et al. (2000) Influence of body composition on the accuracy of reported energy intake in children. Obes Res 8, 597-603.

12. Rennie KL, Jebb SA, Wright A, et al. (2005) Secular trends in under-reporting in young people. Br J Nutr 93, 241-247.

13. Bandini LG, Must A, Cyr H, et al. (2003) Longitudinal changes in the accuracy of reported energy intake in girls 10-15 y of age. Am J Clin Nutr 78, 480-484.

14. Kimm SY, Glynn NW, Obarzanek E, et al. (2006) Racial differences in correlates of misreporting of energy intake in adolescent females. Obesity 14, 156-164.

15. Andersen LF, Pollestad ML, Jacobs DR Jr, et al. (2005) Validation of a pre-coded food diary used among 13-yearolds: comparison of energy intake with energy expenditure. Public Health Nutr 8, 1315-1321.

16. Lioret S, Touvier M, Balin M, et al. (2011) Characteristics of energy under-reporting in children and adolescents. Br J Nutr 105, 1671-1680.

17. Rangan AM, Flood VM \& Gill TP (2011) Misreporting of energy intake in the 2007 Australian Children's Survey: identification, characteristics and impact of misreporters. Nutrients $\mathbf{3}$, 186-199.

18. Murakami K, McCaffrey TA \& Livingstone MBE (2013) Dietary glycaemic index and glycaemic load in relation to food and nutrient intake and indices of body fatness in British children and adolescents. Br J Nutr 110, 1512-1523.

19. Murakami K, Miyake Y, Sasaki S, et al. (2012) Characteristics of under- and over-reporters of energy intake among Japanese children and adolescents: the Ryukyus Child Health Study. Nutrition 28, 532-538.

20. Bandini LG, Cyr H, Must A, et al. (1997) Validity of reported energy intake in preadolescent girls. Am J Clin Nutr 65, Suppl. 4, 1138S-1141S

21. Kontogianni MD, Farmaki AE, Vidra N, et al. (2010) Associations between lifestyle patterns and body mass index in a sample of Greek children and adolescents. J Am Diet Assoc 110, 215-221.

22. Garriguet D (2008) Under-reporting of energy intake in the Canadian Community Health Survey. Health Rep 19, 37-45.
23. Forrestal SG (2011) Energy intake misreporting among children and adolescents: a literature review. Matern Child Nutr 7, 112-127.

24. Moshfegh AJ, Rhodes DG, Baer DJ, et al. (2008) The US Department of Agriculture Automated Multiple-Pass Method reduces bias in the collection of energy intakes. Am J Clin Nutr 88, 324-332.

25. Blanton CA, Moshfegh AJ, Baer DJ, et al. (2006) The USDA Automated Multiple-Pass Method accurately estimates group total energy and nutrient intake. J Nutr 136, 2594-2599.

26. Conway JM, Ingwersen LA, Vinyard BT, et al. (2003) Effectiveness of the US Department of Agriculture 5-step multiple-pass method in assessing food intake in obese and nonobese women. Am J Clin Nutr 77, 1171-1178.

27. Conway JM, Ingwersen LA \& Moshfegh AJ (2004) Accuracy of dietary recall using the USDA five-step multiple-pass method in men: an observational validation study. J Am Diet Assoc 104, 595-603.

28. Zipf G, Chiappa M, Porter KS, et al. (2013) National health and nutrition examination survey: plan and operations, 19992010. Vital Health Stat 1 no. 56, 1-37.

29. Johnson CL, Paulose-Ram R, Ogden CL, et al. (2013) National health and nutrition examination survey: analytic guidelines, 1999-2010. Vital Health Stat 2 no. 161, 1-24.

30. Centers for Disease Control and Prevention, National Center for Health Statistics (2014) National Health and Nutrition Examination Survey. NHANES response rates and population totals. http://www.cdc.gov/nchs/nhanes/response_ rates_cps.htm (accessed December 2014).

31. Centers for Disease Control and Prevention, National Center for Health Statistics (2014) National Health and Nutrition Examination Survey. Questionnaires, datasets, and related documentation. http://www.cdc.gov/nchs/nhanes/ nhanes_questionnaires.htm (accessed December 2014).

32. Kuczmarski RJ, Ogden CL, Guo SS, et al. (2002) 2000 CDC Growth Charts for the United States: methods and development. Vital Health Stat 11 no. 246, 1-190.

33. Centers for Disease Control and Prevention, National Center for Health Statistics (2014) A SAS program for the 2000 CDC Growth Charts (ages 0 to $<20$ years). http://www.cdc.gov/ nccdphp/dnpao/growthcharts/resources/sas.htm (accessed December 2014).

34. Centers for Disease Control and Prevention (2014) Basics about childhood obesity. http://www.cdc.gov/obesity/ childhood/defining.html (accessed December 2014).

35. Black AE (2000) Critical evaluation of energy intake using the Goldberg cut-off for energy intake: basal metabolic rate. A practical guide to its calculation, use and limitations. Int J Obes Relat Metab Disord 24, 1119-1130.

36. Huang TT, Roberts SB, Howarth NC, et al. (2005) Effect of screening out implausible energy intake reports on relationships between diet and BMI. Obes Res 13, 1205-1217.

37. Schofield WN (1985) Predicting basal metabolic rate, new standards and review of previous work. Hum Nutr Clin Nutr 39, Suppl. 1, 5-41.

38. Institute of Medicine (2002) Dietary Reference Intakes for Energy, Carbohydrate, Fiber, Fat, Fatty Acids, Cholesterol, Protein and Amino Acids. Washington, DC: National Academies Press.

39. Centers for Disease Control and Prevention, National Center for Health Statistics (2014) Continuous NHANES web tutorial. http://www.cdc.gov/nchs/tutorials/Nhanes/index_continuous. htm (accessed December 2014).

40. Briefel RR, Sempos CT, McDowell MA, et al. (1997) Dietary methods research in the third National Health and Nutrition 
Examination Survey: underreporting of energy intake. $A m \mathrm{~J}$ Clin Nutr 65, Suppl. 4, 1203S-1209S.

41. Lutomski JE, van den Broeck J, Harrington J, et al. (2011) Sociodemographic, lifestyle, mental health and dietary factors associated with direction of misreporting of energy intake. Public Health Nutr 14, 532-541.

42. Ng SW, Slining MM \& Popkin BM (2014) Turning point for US diets? Recessionary effects or behavioral shifts in foods purchased and consumed. Am J Clin Nutr 99, 609-616.

43. Ogden CL, Carroll MD, Kit BK, et al. (2012) Prevalence of obesity and trends in body mass index among US children and adolescents, 1999-2010. JAMA 307, 483-490.

44. Flegal KM, Carroll MD, Kit BK, et al. (2012) Prevalence of obesity and trends in the distribution of body mass index among US adults, 1999-2010. JAMA 307, 491-497.
45. Ogden CL, Carroll MD, Kit BK, et al. (2014) Prevalence of childhood and adult obesity in the United States, 2011-2012. JAMA 311, 806-814.

46. Mendez MA, Sotres-Alvarez D, Miles DR, et al. (2014) Shifts in the recent distribution of energy intake among U.S. children aged 2-18 years reflect potential abatement of earlier declining trends. J Nutr 144, 1291-1297.

47. Troiano RP, Berrigan D, Dodd KW, et al. (2008) Physical activity in the United States measured by accelerometer. Med Sci Sports Exerc 40, 181-188.

48. Belcher BR, Berrigan D, Dodd KW, et al. (2010) Physical activity in US youth: effect of race/ethnicity, age, gender, and weight status. Med Sci Sports Exerc 42, 2211-2221.

49. Murakami K \& Livingstone MBE (2014) Eating frequency in relation to body mass index and waist circumference in British adults. Int J Obes 38, 1200-1206. 